\title{
The Potential of Dry Winding for Rapid, Inexpensive Manufacture of Composite Overwrapped Pressure Vessels
}

\author{
Andrew Weisberg, Salvador M. Aceves \\ Lawrence Livermore National Laboratory \\ 7000 East Avenue, L-792 \\ Livermore, CA 94551 \\ Phone: (925) 4220864 \\ E-mail: saceves@1lnl.gov
}

\begin{abstract}
We are proposing a methodology for reducing manufacturing time and cost of composite overwrapped pressure vessels (COPVs). Dry winding methodology promises to increase bonding speed enabling rapid vessel winding (10 minutes vs. 3 hours with today's wet winding approach) with no need for oven curing. Faster winding is likely to reduce vessel cost due to higher throughput enabling increased productivity from expensive winding machines as well as reduced labor cost per vessel. Tape manufacture in large scale in a central facility minimizes tape handling and distribution costs. Finally, high ratio of fiber to resin reduces resin cost as well as the wall thickness for any necessary strength, increasing the packaging efficiency of the vessel and reducing weight.
\end{abstract}

\section{Keywords: pressure vessel manufacture, rapid winding}

\section{Introduction}

As a universal transportation fuel that can be generated from water and any energy source, hydrogen $\left(\mathrm{H}_{2}\right)$ is a leading candidate to supplant petroleum with the potential to ultimately eliminate petroleum dependence, associated air pollutants and greenhouse gases [1]. The predominant technical barrier limiting widespread use of hydrogen automobiles is storing enough hydrogen fuel onboard to achieve sufficient (500+ kilometers) driving range in a compact, lightweight, rapidly refuelable, and cost-effective system [2].

Whether at ambient [3-4] or cryogenic temperature [5-6], hydrogen storage in composite overwrapped pressure vessels (COPVs) is necessary for long-range automotive hydrogen propulsion. However, COPVs are expensive and their production processes may be challenged to ramp up fast enough for a large-scale transition to hydrogen-fueled transportation. COPV's rely on filament winding to obtain the full strength of the fiber. Filament winding is a slow, sequential process. New processes that 
can reduce composite manufacturing cost without compromising strength may have broad applicability for reducing costs of COPVs.

In collaboration with industrial partners Quantum and Boeing [7], we have researched methodologies that may contribute to addressing the remaining composite pressure vessel cost efficiency hurdles to enable a rapid transition to hydrogen-fueled automobiles. We propose a process that may offer manufacturing speed and cost advantages independent of the choice of fiber material.

\section{Approach}

In an effort to reduce COPV manufacture cost and time vs. today's wet winding process, we consider the potential for increasing winding speed. Rapid winding has the potential to reduce cost by increasing fiber throughput in expensive vessel manufacturing and curing machines. An important question therefore surfaces: what prevents the proven wet winding composite fabrication processes from being speeded up? Production capital and labor cost components are both inversely proportional to the duration that the part being built spends on a particular piece of equipment. The process that puts all of the costly structure mass into a pressure vessel has intrinsic physical speed limitations.

If one tried to speed up wet winding, process consistency would fall apart. Density variations, fiber not following straight paths, long bubbles between plies, perhaps a large variety of fluidics with insufficient equilibration time would arise with a few-fold speed increase. Substantial (order of magnitude) speedup in wet winding processes is therefore unlikely.

The proposed "Dry Tape" process pays attention to viscous flow and diffusion. It aims at an order of magnitude or more improvement in winding speed - the key rate-limiting manufacturing step. The process is likely to achieve these aims because the viscous flow equations are subsets of the convection-diffusion equations, and diffusion times vary inversely as thickness squared. The target is achieving bond formation on submillisecond timescales.

The proposed dry winding approach may allow considerable cost savings through centralized tape manufacture and rapid winding with no need for oven curing. The technique can be easily scaled up to satisfy the growing demand for inexpensive pressure vessels.

The first step in the dry winding approach consists of making fiber tape at a central facility. Fiber is wetted with resin and then squeezed into rollers, maximizing the ratio of fiber to resin. Preferred resins are thermoplastics as well as some thermosets (epoxy vinyl ester, ring opening metathesis polymerization, isophtalic polyesters) that adhere well and maintain net shapes without oven curing. The resin-impregnated tape is rigid and is therefore sliced into narrow $(\sim 1 \mathrm{~cm})$ strips, enabling it to curve around end dome contours without crumpling. The sliced dry tape is then rolled into reels and transported 
to the vessel winding facility in a refrigerated container. At the vessel production site, the tape is wound at very high speed ( $20 \mathrm{~m} / \mathrm{s}$ vs. $1 \mathrm{~m} / \mathrm{s}$ for today's wet winding processes).

In the future, we envision the use of this procedure for making vessels in a very short time ( 10 minutes vs. 3 hours for wet fiber winding) without the need for oven curing. The proposed two-vendor sequence would be a series processes very similar to electronics manufacturing. The technique can be easily scaled up to satisfy the growing demand for inexpensive pressure vessels.

The proposed vessel winding process has potential for reduced cost vs. today's wet wound vessels due to a synergistic combination of advantages:

- Rapid winding reduces capital cost of winding machine (per kilogram of composite) and labor cost of the machine operator.

- Inter-tape bonding is performed at ambient temperature. No oven curing is necessary.

- High volume production is possible with a single winding machine instead of multiple winding machines and ovens requiring multiple operators.

- High ratio of fiber to resin reduces the cost of resin. It also reduces the wall thickness for any necessary strength, increasing the packaging efficiency of the vessel and reducing weight.

- Tape manufacture in large scale at a central facility minimizes tape handling and distribution costs.

Figure 1 illustrates where the proposed approach lies within all available composite manufacturing process choices, with dry winding tape technology on its rightmost edge. Other efficient approaches to vessel manufacture (wet winding, fiber placement) are being researched by team members Quantum and Boeing as a part of this project. Alternatives at the left of the figure lead to undesirable characteristics due to sacrifice of maximum tensile strength and/or imprecise net shapes.

\section{Results}

The proposed approach combines many of the features of proven high-strength processes, except that it seeks to perform them at high speed to drastically reduce manufacturing cost. Other features and drawbacks of the tape process could affect materials costs and vessel performance, but the effects are likely to be slight and positive. Until the reality of significant manufacturing cost improvements is proven, the investigation of vessel performance effects and potential restrictions on fiber choices would be inconsequential. Therefore we estimated how much the proposed process might save on manufacturing (capital and labor) cost. Figures 2 and 3 break that cost down in a fundamental way, first into what the process equipment adds to manufacturing cost as a function of how much material goes through a costly piece of production equipment how fast (the 'throughput'), and then how much 'throughput' that equipment can achieve when laying down a tape of a particular cross section at a particular speed. Attempts are 
made in these figures to roughly characterize our process vs. the conventional competing process of wet winding.

Although many fiber and matrix material choices should be compatible with this tape innovation, very few provide the confidence to quickly perform our proof of concept experiments. In particular, the repeatability of (rapidly testable) bonding is at risk from airborne contamination and perhaps from humidity. Low material sensitivity to environmental conditions is therefore important. Clean facilities are available to meet these challenges, and plans to procure tape and (slightly hazardous) bonding materials are compatible with standard laboratories' safety and environmental regulations.

\section{Proposed Proof of Concept Experiment}

Demonstration of the technological potential of our approach demands a proof of concept experiment. The proposed proof of concept experiment focuses on determining bonding speed - the key parameter limiting winding velocity. Figure 4 shows a block diagram schematic of the calorimeter designed for a proof of concept test apparatus. This design represents the simplest way to make measurements of a high-speed process without the measuring instrument limiting how fast the process can occur. Its approach relies on detecting the heat being evolved by a tape bonding process to find out how fast that process is occurring, without interfering with the mechanical phenomena that ought to be changing as a bond is forming (as might be the case with speed of sound, ultrasonic impedance, or opacity measurements), or further restricting the choice of experimental gas environment.

Figure 5 shows the key components of the high-speed calorimeter necessary to measure the heat being evolved during the adhesion process. Thermal isolation trusses in the calorimeter retain their dynamic calibration as stuck-together tape test specimens are bonded and taken out of the instrument.

\section{Conclusions}

We propose a methodology for reducing manufacturing time and cost of composite overwrapped pressure vessels (COPVs). Dry winding methodology promises to increase bonding speed enabling rapid vessel winding (10 minute vs. 3 hours with today's wet winding approach) with no need for oven curing. The proposed approach is the subject of a recent patent [8].

Cost reduction is likely due to higher throughput enabling increased productivity from expensive winding machines as well as reduced labor cost per vessel. Tape manufacture in large scale in a central facility minimizes tape handling and distribution costs. Finally, high ratio of fiber to resin reduces resin cost as well as the wall thickness for any necessary strength, increasing the packaging efficiency of the vessel and reducing weight. 
A proof of concept experiment is necessary for demonstrating rapid (sub millisecond) bonding - the key parameter enabling rapid vessel manufacture. The approach selected demands construction of a calorimeter to detect heat evolved during the adhesion process.

\section{Acknowledgments}

This project was funded by DOE, Office of Fuel Cell Technologies, Jesse Adams, Technology Development Manager. This work performed under the auspices of the U.S. Department of Energy by Lawrence Livermore National Laboratory under Contract DEAC52-07NA27344.

\section{References}

1. Berry GD, Aceves SM. The case for hydrogen in a carbon constrained world. Journal of Energy Resources Technology, 2005;127:89-94.

2. Berry GD, Martinez-Frias, J, Espinosa-Loza, F, Aceves SM. Hydrogen Storage and Transportation. Encyclopedia of Energy, 2005;3:267-281.

3. Irani RS. Hydrogen Storage: High pressure gas containment. MRS Bulletin, 2002;27:680-682.

4. Barthelemy H. Hydrogen Storage - Industrial perspectives. International Journal of Hydrogen Energy, 2012;37:17364-17372.

5. Aceves SM, Petitpas G, Espinosa-Loza F, Matthews MJ, Ledesma-Orozco E. Safe, long range, inexpensive and rapidly refuelable hydrogen vehicles with cryogenic pressure vessels. International Journal of Hydrogen Energy, 2013;38:2480-89.

6. Petitpas G, Benard P, Klebanoff LE, Xiao J, Aceves S. A Comparative Analysis of the Cryo-Compression and Cryo-Adsorption Hydrogen Storage Methods, International Journal of Hydrogen Energy, 2014;39:10564-10584.

7. Leavitt M. Development of advanced manufacturing technologies for low cost hydrogen storage vessels. FY2013 Annual Progress Report, DOE Hydrogen and Fuel Cells Program, 2013;VI: 30-34.

8. Weisberg AH. Methods for tape fabrication of continuous filament composite parts and articles of manufacture thereof. United States Patent US 8545657 B2, 2013. 


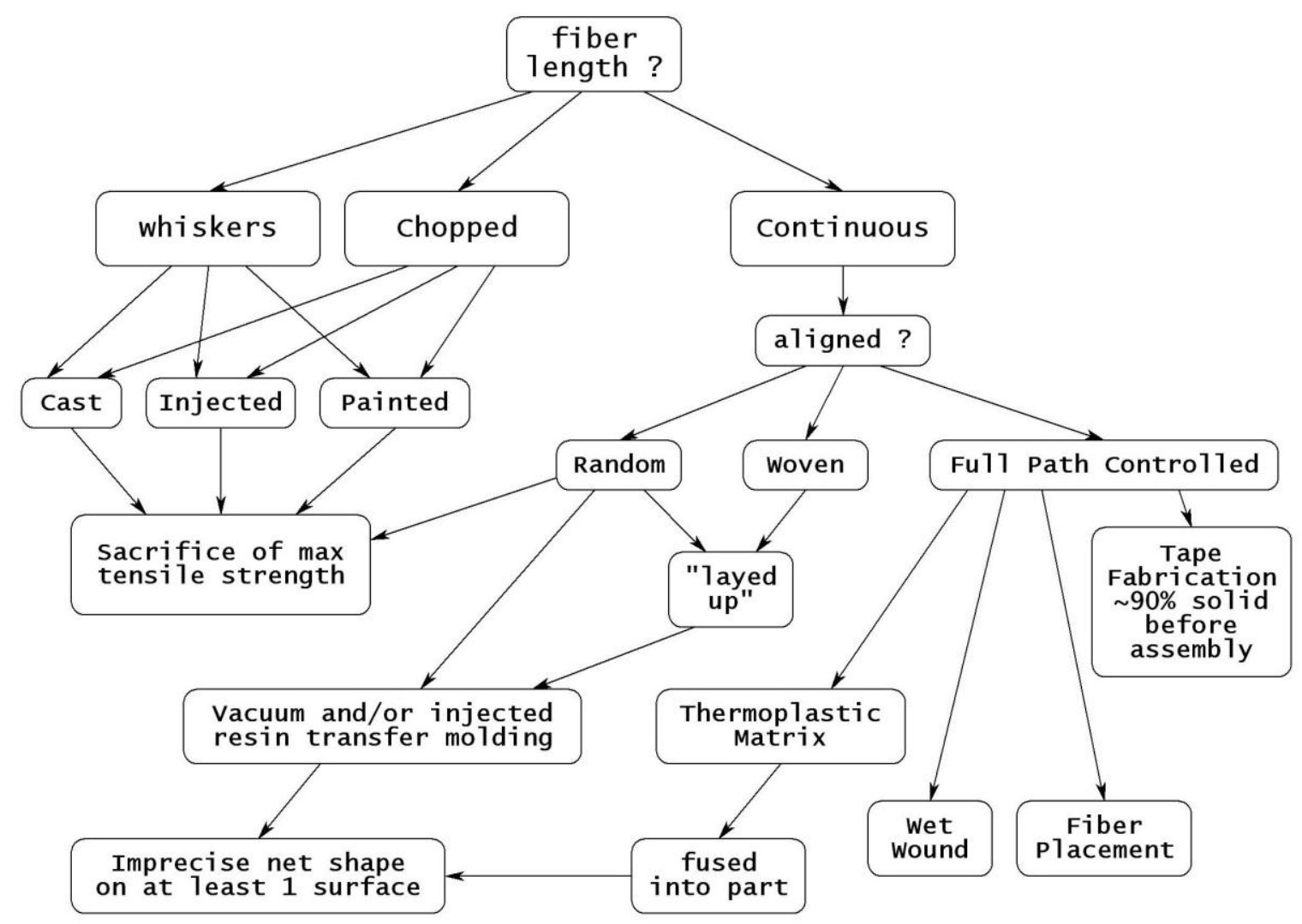

Figure 1. Bubble diagram dissects the various options for manufacturing composite vessels, starting from choices of the geometry of the individual composite fibers. The proposed approach, tape fabrication, occupies the extreme right in the diagram. 


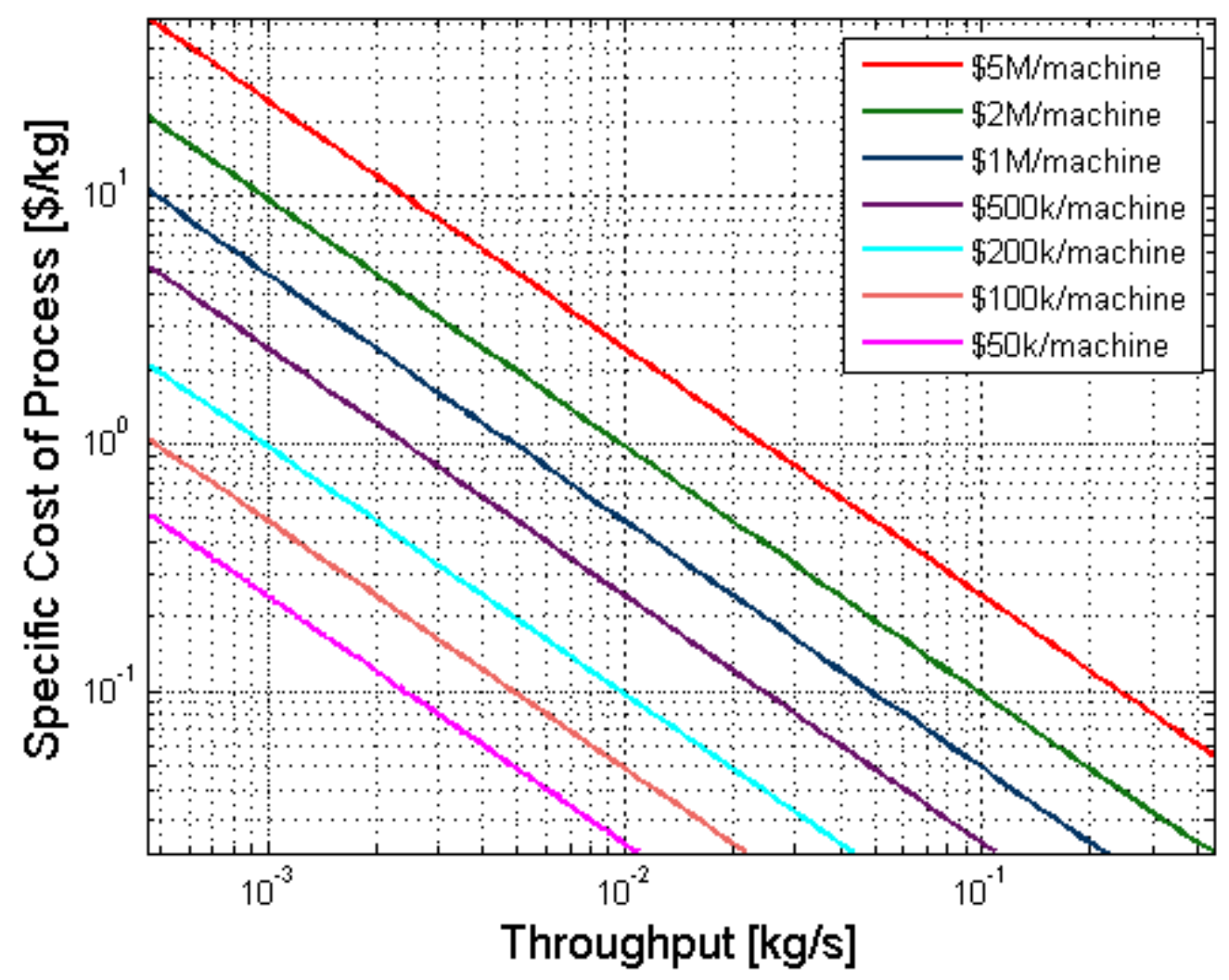

Figure 2. Cost to perform a composite manufacturing process versus the composite material processing rate ('throughput' in units of mass-per-unit-time), at various levels of production capital cost (presuming a 3 year return on investment). The proposed dry tape process projects operation in the bottom right edge of this graph, while conventional wet winding processes $(0.02 \mathrm{~kg} / \mathrm{s})$ land roughly in the center of the figure. 


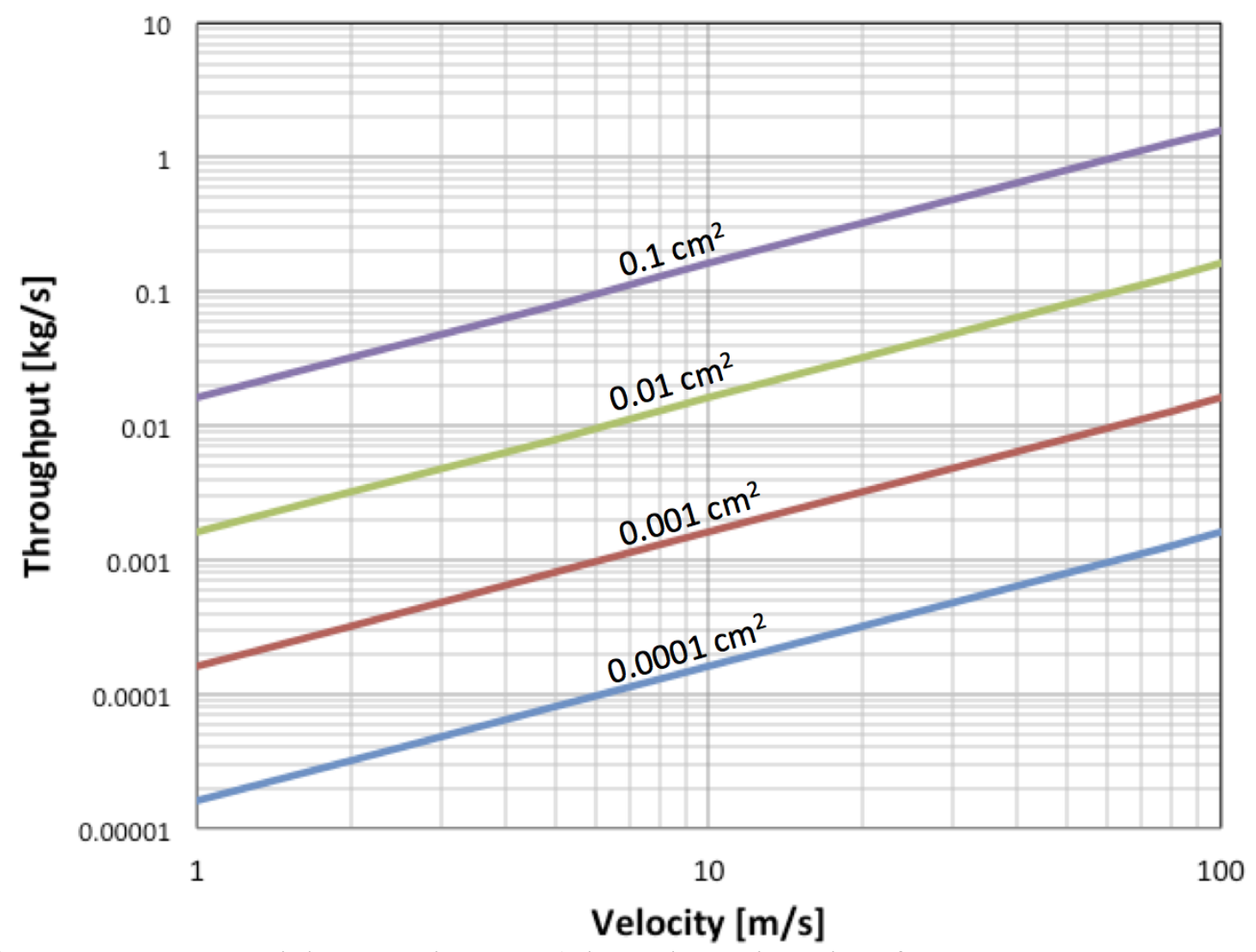

Figure 3. Tape material processing rate ('throughput' in units of mass-per-unit-time) as a function of tape velocity, at various levels of tape cross sectional area. Current wet winding processes occupy the middle of the left edge $(1 \mathrm{~m} / \mathrm{s}$ and $0.02 \mathrm{~kg} / \mathrm{s})$. Dry tape winding would maintain or increase cross sectional area while increasing winding speed to $\sim 20 \mathrm{~m} / \mathrm{s}$. 


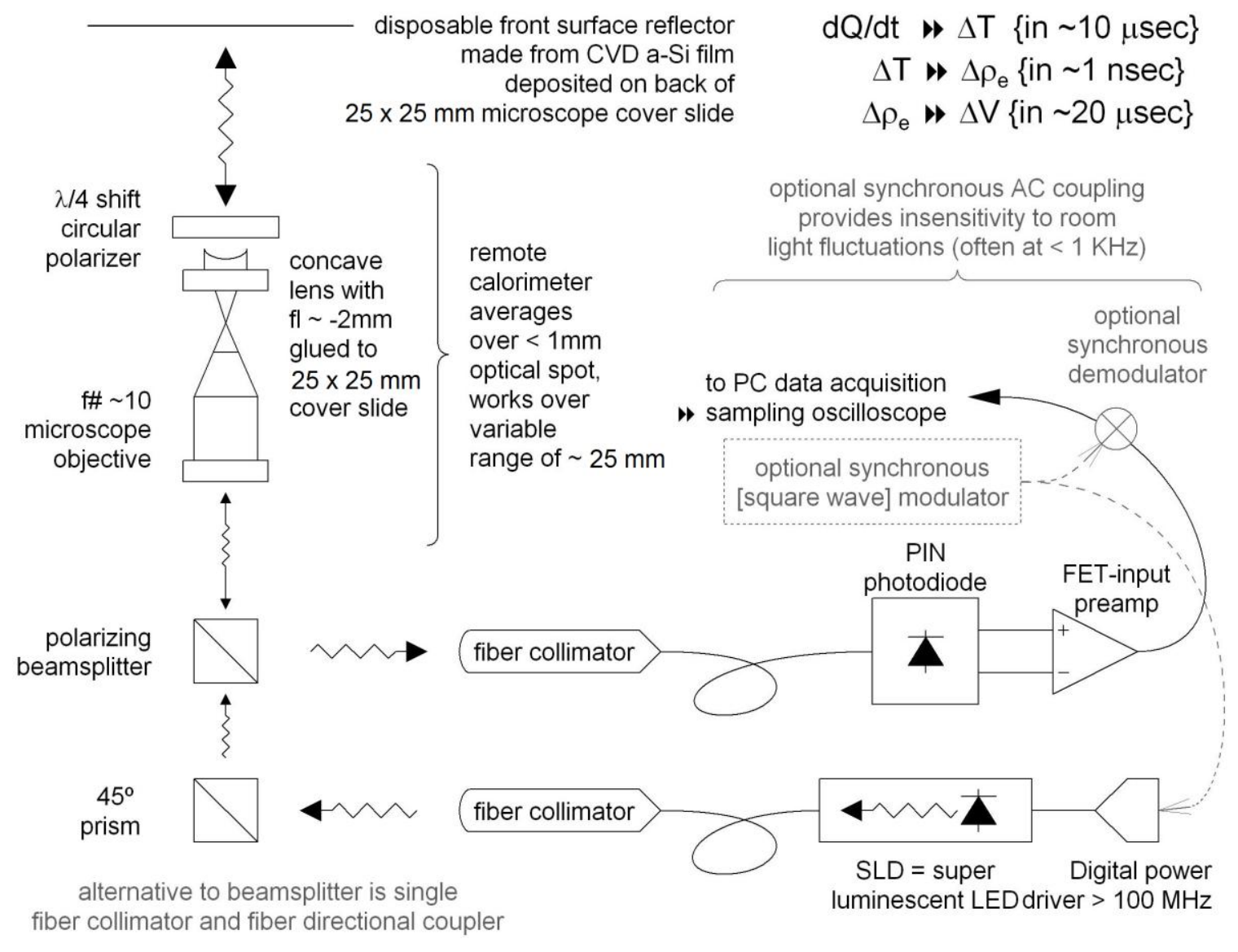

Figure 4. Schematic diagram of calorimeter to determine tape bonding speed by capturing temperature rise due to bonding with a digital oscilloscope. This hardware diagram mixes physical components, optical beam paths including captured photons in fibers, and electronics. 


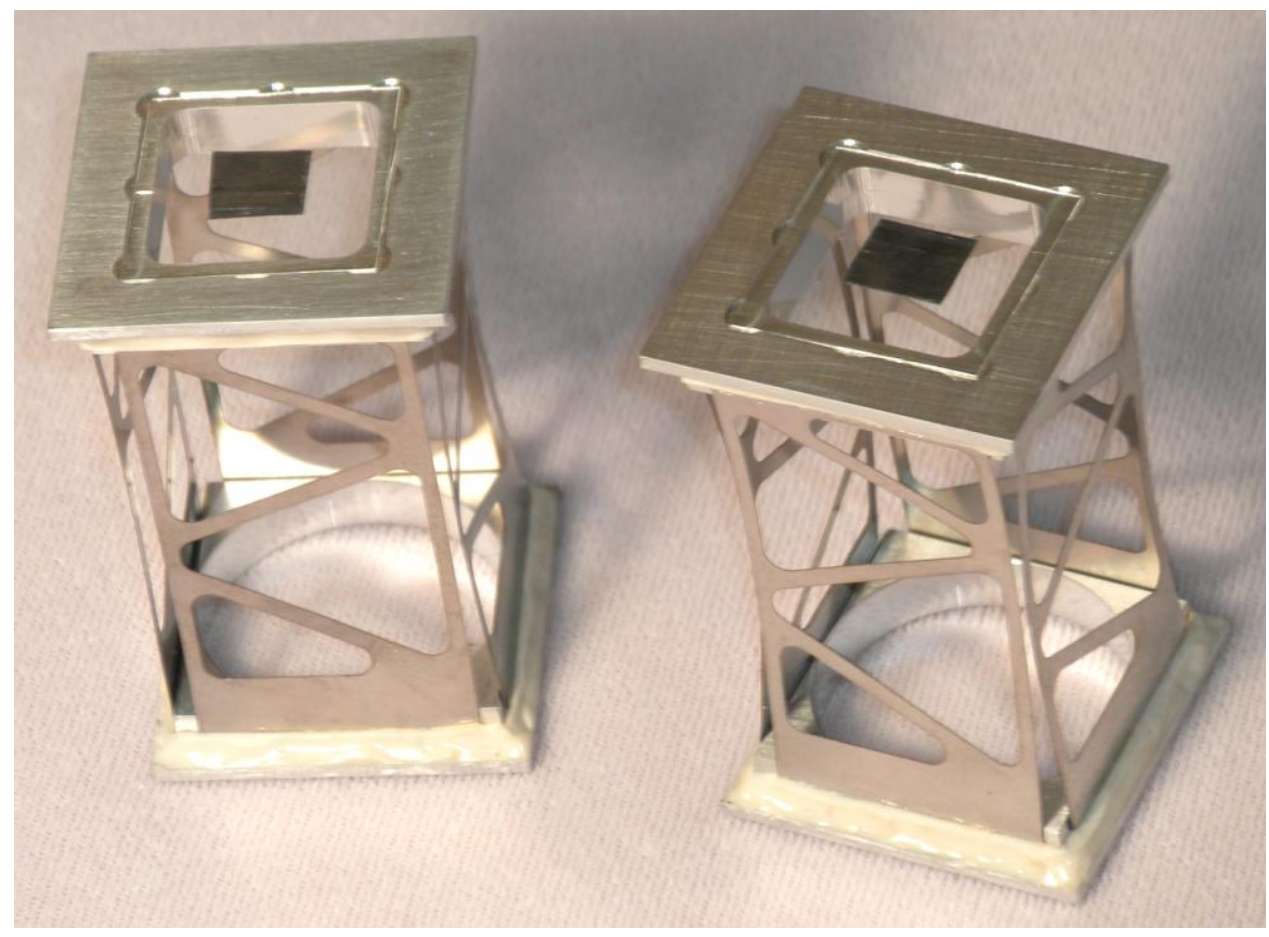

Figure 5. Thermal isolation truss components for proof of concept experiment. These truss stages mount tape specimens (black squares in the center of clear microscope cover slides, glued atop these trusses). One of these trusses is inverted and its tape specimen placed in abrupt contact with the other tape specimen to determine bonding speed with the calorimeter apparatus of Figure 4. 